Pacific Journal of Mathematics

ON HYPOELLIPTIC DIFFERENTIAL OPERATORS OF 


\title{
ON HYPOELLIPTIC DIFFERENTIAL OPERATORS OF CONSTANT STRENGTH
}

\author{
M. ShafiI-MOUSAVI AND Z. ZielezNy
}

A hypoelliptic differential operator $P(D)$ with constant coefficients has the following property: For every $u \in \mathscr{D}^{\prime}(\Omega)$, if $P(D) u$ is in a Gerrey class in $\Omega$ then so is $u$ (though the two Gevrey classes are not necessarily the same).

In this paper we prove that hypoelliptic differential operators with variable coefficients have locally the same property, if they are of constant strength and their coefficients are in a Gevrey class.

Let $P(x, D)$ be a differential operator with $C^{\infty}$ coefficients and of constant strength in a neighborhood of a point $x^{\circ} \in R^{n}$. L. Hörmander ([1], Theorem 7.3.1) proved the following local existence theorem:

There exists a sufficiently small neighborhood $\Omega$ of $x^{\circ}$ and a linear mapping $E$ of $\mathscr{E}^{\prime}\left(R^{n}\right)$ into $\mathscr{E}^{\prime}\left(R^{n}\right)$ with the properties

$$
\begin{array}{llll}
P(x, D) E u=u \text { in } \Omega, & \text { if } & & u \in \mathscr{E}^{\prime}\left(R^{n}\right), \\
E P(x, D) v=v \text { in } \Omega, & \text { if } & v \in \mathscr{E}^{\prime}(\Omega),
\end{array}
$$

and

$$
\|E u\|_{p, \tilde{P}_{0} k} \leqq C_{k}\|u\|_{p, k}, \quad \text { if } \quad u \in \mathscr{E}^{\prime}\left(R^{n}\right) \cap \mathscr{B}_{p, k}, \quad k \in \mathscr{K} .
$$

If, in addition, the operator $P_{\circ}(D)=P\left(x^{\circ}, D\right)$ is hypoelliptic, then every solution $u \in \mathscr{D}^{\prime}(\Omega)$ of the equation

$$
P(x, D) u=v
$$

is in $C^{\infty}(\Omega)$, whenever $v \in C^{\infty}(\Omega)$ (see [1], Theorem 7.4.1).

But $P_{\circ}(D)$, being hypoelliptic, is $d$-hypoelliptic for some $d \geqq 1$, i.e., every solution $u \in \mathscr{D}^{\prime}(\Omega)$ of the equation

$$
P_{\circ}(D) u=v
$$

is in the Gevrey class $\Gamma^{\rho^{\prime}}(\Omega)$, when $v \in \Gamma^{\circ}(\Omega)$ and $\rho^{\prime}=\max \{d, \rho\}$. The question arises whether the same is true for equation (4), if the coefficients of $P(x, D)$ are in $\Gamma^{\circ}(\Omega)$.

In this paper we prove a theorem on Gevrey regularity for a very wide class of operators-not necessarily differential operators. Next we apply this general theorem to a differential operator $P(x, D)$ of constant strength in a neighborhood of $x^{\circ} \in R^{n}$. We choose $\Omega$ as in the existence theorem mentioned above. If the coefficients of $P(x, D)$ are in $\Gamma^{\circ}(\Omega)$ and $P_{\circ}(D)$ is $d$-hypoelliptic, we show that every solution 
$u \in \mathscr{E}^{\prime}(\Omega)$ of equation (4) is in $\Gamma^{d \rho^{\prime}}(\Omega)$, when $v \in \Gamma^{\rho}(\Omega) \cap \mathscr{E}^{\prime}(\Omega)$ and $\rho^{\prime}=\max \{d, \rho\}$.

Throughout the paper we use the notation of [1] and [3].

1. The general theorem. We recall that, if $\Omega$ is an open subset of $R^{n}$ and $\rho>0$, the $\rho$ th Gevrey class $\Gamma^{\rho}(\Omega)$ can be defined as the set of all functions $u \in C^{\infty}(\Omega)$ with the property: To every compact set $K \subset \Omega$ there exists a constant $A$ (depending on $K$ and $u$ ) such that

$$
\left\|D^{\alpha} u\right\|_{K} \leqq A^{|\alpha|+1}(|\alpha| !)^{\rho},
$$

where \|\|$_{K}$ is the $L^{2}$-norm over $K$ and $\alpha$ is any multi-index.

Similarly, if $P$ is a polynomial in $R^{n}$ of degree $m$, we denote by $\Gamma_{P}^{\circ}(\Omega)$ the set of all functions $u \in C^{\infty}(\Omega)$ with the property: To every compact set $K \subset \Omega$ there exists a constant $B$ (depending on $P, K$ and u) such that

$$
\left\|P^{j}(D) u\right\|_{K} \leqq B^{j+1}(j !)^{m \rho}, \quad j=0,1, \cdots,
$$

where $P^{j}(D)$ is the $j$ th power of $P(D)$.

The polynomial $P$ is $d$-hypoelliptic if and only if there is a constant $C$ such that, for every $\alpha$,

$$
|\xi|^{|\alpha| / d}\left|D^{\alpha} P(\xi)\right| \leqq C(1+|P(\xi)|), \quad \xi \in R^{n},
$$

(see [4], p. 440). Hence it follows that

$$
|\xi|^{2} \leqq C^{\prime}\left(1+|P(\xi)|^{2}\right)^{d / m}
$$

and

$$
\left|D^{\alpha} P(\xi)\right| \leqq C^{\prime \prime}(1+|P(\xi)|)^{1-|\alpha| / m d}, \quad \xi \in R^{n},
$$

where $m$ is the degree of $P$ and $C^{\prime}, C^{\prime \prime}$ are constants.

The following theorem is a refined version of a theorem stated in [3]; we include the proof for the sake of completeness.

THEOREM 1. If $P$ is a d-hypoelliptic polynomial then, for any open set $\Omega \subset R^{n}$ and any $\rho>0$, we have

$$
\Gamma_{P}^{\rho}(\Omega) \subset \Gamma^{d \rho^{\prime}}(\Omega),
$$

where $\rho^{\prime}=\max \{d, \rho\}$

Proof. We may assume that $d$ is the smallest number for which the estimate (5) holds. Then $d$ is a rational number and we can write $d / m=\mu / \nu$, where $\mu$ and $\nu$ are integers $>0$. It follows that

$$
\left|\xi^{\alpha}\right|^{2} \leqq C_{1}\left(1+\left|P^{\mu}(\xi)\right|^{2}\right), \quad \xi \in R^{n},
$$


for some constant $C_{1}$ and every $\alpha$ with $|\alpha| \leqq \nu$. Furthermore, the polynomial $P^{\mu}$ is $d$-hypoelliptic, and so

$$
\left|D^{\alpha} P^{\mu}(\xi)\right| \leqq C_{2}\left(1+\left|P^{\mu}(\xi)\right|\right)^{1-|\alpha| / m \mu d}, \quad \xi \in R^{n},
$$

by (6).

Let $\Omega$ be a bounded open subset of $R^{n}$ and $\Omega_{\delta}, \delta>0$, the set of points $x \in \Omega$ whose distance from $C \Omega$ is greater than $\delta$. By a theorem of Hörmander ([2], Theorem 4.2), conditions (7) and (8) imply that, for every $s \geqq 0$ and $t>0$,

$$
\sup _{0<\tau \leqq t} \tau^{\lambda}\left\|D^{\alpha} v\right\|_{\Omega_{s+\tau}} \leqq C\left\{\sup _{0<\tau \leqq t} \tau^{\lambda}\left\|P^{\mu}(D) v\right\|_{\Omega_{s+\tau}}+\|v\|_{\Omega_{s}}\right\}, \quad v \in C^{\infty}\left(\Omega_{s}\right),
$$

where $|\alpha| \leqq \nu, \lambda=$ mpd and $C$ is a constant depending only on $P$, $\mu, \nu$, and the diameter of $\Omega$. Hence

$$
\left\|D^{\alpha} v\right\|_{\Omega_{s+t}} \leqq C\left\{\left\|P^{\mu}(D) v\right\|_{\Omega_{s}}+t^{-\lambda}\|v\|_{\Omega_{s}}\right\}, \quad v \in C^{\infty}\left(\Omega_{s}\right) .
$$

Given an integer $k \geqq 1$ and a $\delta>0$, we apply (9) with $s=(1-1 / k)^{\delta}$, $t=\delta / k$ and $v=D^{\beta} u$, where $|\beta|=(k-1) \nu$. Next (if $k>1$ ) we apply again (9) to both terms on the right-hand side with $s=(1-2 / k) \delta$, $t=\delta / k$ and either $v=D^{r} P^{\mu}(D)$ or $v=D^{\gamma} u$, where $|\gamma|=(k-2) \nu$. After $k$ such steps we obtain

(10) $\max _{|\alpha|=k \nu}\left\|D^{\alpha} u\right\|_{\Omega_{\delta}} \leqq C^{k} \sum_{i=0}^{k}\left(\begin{array}{c}k \\ i\end{array}\right)\left(\frac{k}{\delta}\right)^{\lambda i}\left\|P^{(k-i) \mu}(D) u\right\|_{\Omega}, \quad u \in C^{\infty}(\Omega)$.

On the other hand, for each multi-index $\alpha$ with $|\alpha| \leqq \nu-1$ there is a multi-index $\beta$ with $|\beta|=\nu$ such that

$$
\left|\xi^{\alpha}\right|^{2} \leqq 1+\left|\xi^{\beta}\right|^{2}, \quad \xi \in R^{n} .
$$

Therefore, by the same theorem of Hörmander, there is a constant $C^{\prime}$ such that

$$
\left\|D^{\alpha} u\right\|_{\Omega_{2 \delta}} \leqq C^{\prime}\left\{\max _{|\beta|=\nu}\left\|D^{\beta} u\right\|_{\Omega_{\delta}}+\|u\|_{\Omega_{\delta}}\right\}, \quad u \in C^{\infty}\left(\Omega_{\delta}\right),
$$

where $|\alpha| \leqq \nu-1$; the constant $C^{\prime}$ may depend on $\delta$.

If $\alpha$ is an arbitrary multi-index and $k \nu \leqq|\alpha|<(k+1) \nu$, we choose another multi-index $\beta$ so that $|\alpha-\beta| \leqq \nu-1$ and $|\beta|=k \nu$. Then condition (11) yields

$$
\left\|D^{\alpha} u\right\|_{\Omega_{2 \delta}} \leqq C^{\prime}\left\{\max _{|\beta|=(\nu+1) k}\left\|D^{\beta} u\right\|_{\Omega_{\delta}}+\max _{|\beta|=\nu k}\left\|D^{\beta} u\right\|_{\Omega_{\delta}}\right\}, \quad u \in C^{\infty}\left(\Omega_{\tilde{o}}\right) .
$$

Combining this estimate with (10), we obtain 


$$
\begin{gathered}
\left\|D^{\alpha} u\right\|_{\Omega_{2 \delta}} \leqq C^{\prime} C^{k+1} \sum_{i=0}^{k+1}\left(\begin{array}{c}
k+1 \\
i
\end{array}\right)\left(\frac{k+1}{\delta}\right)^{\lambda i}\left\|P^{(k+1-i) \mu}(D) u\right\|_{\Omega} \\
+C^{\prime} C^{k} \sum_{i=0}^{k}\left(\begin{array}{c}
k \\
i
\end{array}\right)\left(\frac{k}{\delta}\right)^{\lambda i}\left\|P^{(k-i) \mu}(D) u\right\|_{\Omega} .
\end{gathered}
$$

Suppose now that $u \in \Gamma_{P}^{o}\left(\Omega^{\prime}\right)$, where $\Omega^{\prime}$ is an arbitrary open set in $R^{n}$. If $K$ is a compact subset of $\Omega^{\prime}$, there exists a bounded open set $\Omega$ and a $\delta>0$ such that $K \subset \Omega_{2 \delta} \subset \Omega \subset \subset \Omega^{\prime}$. It follows that, for some constant $B$,

$$
\left\|P^{i}(D) u\right\|_{\Omega} \leqq B^{i+1} i^{i m \rho}, \quad i=0,1, \cdots .
$$

Note that in the definition of $\Gamma_{P}^{\rho}(\Omega)$ the terms $(j !)^{m \rho}$ can be replaced by $j^{j m \rho}$, by the Stirling formula. This leads to

$$
\begin{aligned}
k^{i \lambda}\left\|P^{(k-i)} P(D) u\right\|_{\Omega} & \leqq\left[(B+1) \mu^{m \rho}\right]^{k+1} k^{k m \mu \rho+i\left(\lambda-m !{ }^{\prime} \rho\right)} \\
& \leqq\left[(B+1) \mu^{m \rho}\right]^{k+1} k^{k m \mu \rho^{\prime}}
\end{aligned}
$$

$i=0,1, \cdots, k$, where $\rho^{\prime}=\max \{d, \rho\}$. Applying the estimates (13) to the righ-hand side of (12) we can find two constants $B_{1}$ and $B_{2}$ such that

$$
\begin{aligned}
\left\|D^{\alpha} u\right\|_{\Omega_{2 \delta}} \leqq B_{1}^{k+1} k^{k m \mu \rho^{\prime}} & \leqq B_{2}^{|\alpha|+1}|\alpha|^{|\alpha| m \mu \rho^{\prime} / \nu} \\
& =B_{2}^{|\alpha|+1}|\alpha|^{|\alpha| \alpha \rho^{\prime}}
\end{aligned}
$$

Thus $u \in I^{d \rho^{\prime}}\left(\Omega^{\prime}\right)$ and the proof is complete.

We are now in a position to prove the general theorem. We denote by \|\| the $L^{2}$-norm over $R^{n}$.

THEOREM 2. Let $\Omega$ be an open subset of $R^{n}$ and let $T$ be a mapping of $C_{\circ}^{\infty}(\Omega)$ into $C_{\circ}^{\infty}(\Omega)$ whose powers $T^{j}, j=1,2, \cdots$, have the following properties:

$\left(t_{1}\right)$ There exists a d-hypoelliptic polynomial $P$ such that

$$
\left\|P^{j}(D) u\right\| \leqq A^{j}\left\|T^{j} u\right\|, \quad u \in C_{\circ}^{\infty}(\Omega), \quad j=1,2, \cdots,
$$

where $A$ is a constant independent of $j$.

$\left(t_{2}\right)$ For some $\rho>1$,

$$
\left\|T^{j} v\right\| \leqq B^{j+1}(j !)^{m \rho}, \quad v \in \Gamma^{\rho}(\Omega) \cap \mathscr{E}^{\prime}(\Omega), \quad j=1,2, \cdots,
$$

where $m$ is the degree of $P$ and $B$ is a constant independent of $j$.

Then every solution $u \in C_{\circ}^{\infty}(\Omega)$ of the equation

$$
T u=v
$$

is in $\Gamma^{d \rho^{\prime}}(\Omega)$, when $v \in \Gamma^{\rho}(\Omega) \cap \mathscr{E}^{\prime}(\Omega)$; here, as before, $\rho^{\prime}=\max \{d, \rho\}$. 
Proof. The theorem follows immediately from Theorem 1 . In fact, let $u \in C_{\circ}^{\infty}(\Omega)$ be a solution of equation (14) with $v \in \Gamma^{\circ}(\Omega) \cap$ $\mathscr{E}^{\prime}(\Omega)$. Making use of conditions $\left(t_{1}\right)$ and $\left(t_{2}\right)$ we then have

$$
\begin{aligned}
\left\|P^{j}(D) u\right\| & \leqq A^{j}\left\|T^{j} u\right\| \\
& =A^{j}\left\|T^{j-1} v\right\| \leqq(A B)^{j}(j !)^{m \rho}, \quad j=1,2, \cdots .
\end{aligned}
$$

This proves that $u \in \Gamma_{P}^{\rho}(\Omega)$. Since $P$ is $d$-hypoelliptic, it follows that $u \in \Gamma^{d \rho^{\prime}}(\Omega)$, by Theorem 1 .

2. Differential operators of constant strength. The following lemma is essential for the proof of our next theorem.

Lemma. Let $P_{\circ}$ be a hypoelliptic polynomial of degree $m$ and set $k=\widetilde{P}_{0}$. Then

$$
k_{\nu}(\xi)=\sup _{|\eta| \leqq \nu} k(\xi+\eta), \quad \nu>0,
$$

is a function in $\mathscr{K}$ with the properties

$$
\begin{gathered}
k(\xi) \leqq k_{\nu}(\xi) \leqq k(\xi)(1+C \nu)^{m}, \quad \xi \in R^{n}, \\
\left(k^{j}\right)_{\nu}=\left(k_{\nu}\right)^{j}, \\
M_{k_{\nu}}(\xi)=\sup _{\xi^{\prime}} \frac{k_{\nu}\left(\xi+\xi^{\prime}\right)}{k_{\nu}\left(\xi^{\prime}\right)} \leqq(1+C|\xi|)^{m}, \quad \xi \in R^{n},
\end{gathered}
$$

where $C$ is a constant, and

(19) $\quad M_{k_{\nu}} \rightarrow 1$ uniformly on compact subsets of $R^{n}$ as $\nu \rightarrow \infty$.

Proof. The properties (16)-(18) follow immediately from (15) and the inequality

$$
k(\xi+\eta) \leqq k(\xi)(1+C|\eta|)^{m},
$$

which is valid for $k=\widetilde{P}_{\circ}$. We prove the convergence (19).

If $|\xi| \leqq \mu$, then

$$
\frac{k_{\nu}\left(\xi+\xi^{\prime}\right)}{k_{\nu}\left(\xi^{\prime}\right)} \leqq \frac{\sup _{|\eta| \leq \mu+\nu} k\left(\xi^{\prime}+\eta\right)}{\sup _{|\eta| \leqq \nu} k\left(\xi^{\prime}+\eta\right)} .
$$

We claim that the right-hand side of (20), which is $\geqq 1$, converges to 1 as $\nu \rightarrow \infty$, uniformly for $\xi^{\prime} \in R^{n}$.

Assume the contrary. Then there exist $\varepsilon>0$, a sequence $\left\{\nu_{j}\right\} \subset R$, $\nu_{j} \rightarrow \infty$, and sequences $\left\{\xi_{j}^{\prime}\right\},\left\{\eta_{j}\right\},\left\{\eta_{j}^{\prime}\right\} \subset R^{n}$ such that

$$
k\left(\xi_{j}^{\prime}+\eta_{j}\right)=\sup _{|\eta| \leqq \mu+\nu_{j}} k\left(\xi_{j}^{\prime}+\eta\right), \quad k\left(\xi_{j}^{\prime}+\eta_{j}^{\prime}\right)=\sup _{|\eta| \iota_{j}} k\left(\xi_{j}^{\prime}+\eta\right),
$$


and

$$
\frac{k^{2}\left(\xi_{j}^{\prime}+\eta_{j}\right)}{k^{2}\left(\xi_{j}^{\prime}+\eta_{j}^{\prime}\right)} \geqq 1+\varepsilon,
$$

Obviously we have

$$
\nu_{j}<\left|\eta_{j}\right| \leqq \mu+\nu_{j}
$$

and

$$
k\left(\xi_{j}^{\prime}+\eta_{j}\right) \geqq k\left(\xi_{j}^{\prime}-\eta_{j}\right),
$$$$
j=1,2, \cdots .
$$

But, for each $j$, one of the inequalities

$$
\left|\xi_{j}^{\prime}+\eta_{j}\right|>\nu_{j} \text { or }\left|\xi_{j}^{\prime}-\eta_{j}\right|>\nu_{j}
$$

must be satisfied. For, otherwise we could write

$$
2\left|\eta_{j}\right|=\left|\left(\xi_{j}^{\prime}+\eta_{j}\right)-\left(\xi_{j}^{\prime}-\eta_{j}\right)\right| \leqq\left|\xi_{j}^{\prime}+\eta_{j}\right|+\left|\xi_{j}^{\prime}-\eta_{j}\right| \leqq 2 \nu_{j},
$$

which contradicts the first inequality in (23). Since $P_{\circ}$ is a hypoelliptic polynomial, it follows from (24) and (25) that $\left|\xi_{j}^{\prime}+\eta_{j}\right| \rightarrow \infty$.

On the other hand, if $\nu_{j}>\mu / 2$, then

$$
\frac{k^{2}\left(\xi_{j}^{\prime}+\eta_{j}\right)}{k^{2}\left(\xi_{j}^{\prime}+\eta_{j}-\mu \frac{\eta_{j}}{\left|\eta_{j}\right|}\right)} \geqq 1+\varepsilon,
$$

in view of (21), (22) and (23). Furthermore, since $k^{2}$ is a polynomial of degree $2 m$,

$$
k^{2}\left(\xi_{j}^{\prime}+\eta_{j}-\mu \frac{\eta_{j}}{\left|\eta_{j}\right|}\right) \geqq k^{2}\left(\xi_{j}^{\prime}+\eta_{j}\right)-\sum_{\alpha \neq 0}\left|D^{\alpha} k^{2}\left(\xi_{j}^{\prime}+\eta_{j}\right)\right| \frac{(1+\mu)^{2 m}}{\alpha !},
$$

by Taylor's formula. But we assumed that the polynomial $P_{\circ}$ is hypoelliptic and therefore

$$
\frac{D^{\alpha} P_{\circ}(\xi)}{P_{\circ}(\xi)} \longrightarrow 0, \text { if } \alpha \neq 0 \text { and }|\xi| \longrightarrow \infty
$$

(see [1], p. 99, Theorem 4.1.3). Hence it follows that

$$
\frac{D^{\alpha} k^{2}(\xi)}{k^{2}(\xi)} \longrightarrow 0 \text {, if } \alpha \neq 0 \text { and }|\xi| \longrightarrow \infty \text {. }
$$

If now $\left|\xi_{j}^{\prime}+\eta_{j}\right|$ is sufficiently large, we obtain from (27) the estimates

$$
k^{2}\left(\xi_{j}^{\prime}+\eta_{j}-\mu \frac{\eta_{j}}{\left|\eta_{j}\right|}\right) \geqq k^{2}\left(\xi_{j}^{\prime}+\eta_{j}\right)\left(1-\frac{\varepsilon}{2}\right),
$$

which contradict the inequalities (26), when $\varepsilon<1$. 
The contradiction proves the desired convergence of the righthand side of (20). The property (19) is an immediate consequence of that fact.

We remark that the weight function $k_{\delta}$ defined in [1] (see p. 35, Theorem 2.1.2) cannot be used for our purpose.

THEOREM 3. Let $P(x, D)$ be a differential operator of constant strength in a neighborhood of $x^{\circ}$ and let $\Omega$ be a sufficiently small open neighborhood of $x^{\circ}$ for which the local existence theorem is valid. If the coefficients of $P(x, D)$ are in $\Gamma^{\rho}(\Omega), \rho>1$, and if the operator $P_{\circ}(D)=P\left(x^{\circ}, D\right)$ is d-hypoelliptic, then every solution $u \in$ $\mathscr{E}^{\prime}(\Omega)$ of equation (4) is in $\Gamma^{d \rho^{\prime}}(\Omega)$, whenever $\nu \in \Gamma^{\circ}(\Omega) \cap \mathscr{E}^{\prime}(\Omega)$.

Proof. Since $P(x, D)$ is of constant strength in $\Omega$ and $P_{\circ}(D)$ is hypoelliptic, every solution $u \in \mathscr{E}^{\prime}(\Omega)$ of equation (4) is in $C_{0}^{\infty}(\Omega)$, whenever $v \in C_{0}^{\infty}(\Omega)$ (see [1], p. 176, Theorem 7.4.1). For the proof of Theorem 3 it now suffices to show that $P(x, D)$ satisfies the conditions imposed on $T$ in Theorem 2 .

We first observe that, by the lemma, we can replace in the proof of the local existence theorem the function $k_{\delta}$ by our function $k_{\nu}$. Next we recall that condition (3) is derived from the estimate

$$
\|E f\|_{p, \tilde{P}_{\circ} k_{\delta}} \leqq C_{\circ}\|f\|_{p, k_{\delta}} \quad f \in \mathscr{E}^{\prime}\left(R^{n}\right) \cap \mathscr{B}_{p, k},
$$

where $C_{\circ}$ is a constant independent of $f, k$ and $\delta$ (see [1], p. 176). Hence, if $p=2, k=\widetilde{P}_{\circ}$ and $k_{\nu}$ is as in our lemma, we obtain

$$
\left\|E^{j} u\right\|_{2, k\left(k^{j-1}\right)_{\nu}} \leqq A\left\|E^{j-1} u\right\|_{2,\left(k^{j-1}\right)_{\nu}}, \quad u \in C_{\circ}^{\infty}(\Omega),
$$

where $A$ is a constant independent of $j$ and $u$. But, in view of (16) and (17), there is a constant $B$ independent of $j$ and $u$ such that

$$
\left\|E^{j-1} u\right\|_{2,\left(k^{j-1}\right)_{\nu}} \leqq B\left\|E^{j-1} u\right\|_{2, k\left(k^{j-2}\right)_{\nu}}, \quad u \in C_{\circ}^{\infty}(\Omega) .
$$

Condition (16) and repeated application of (28) and (29) gives

$$
\left\|E^{j} u\right\|_{2, k^{j}} \leqq\left\|E^{j} u\right\|_{2, k\left(k^{j}-1\right)_{\nu}} \leqq(A B)^{j}\|u\|, \quad u \in C_{\circ}^{\infty}(\Omega) .
$$

Substituting now $P(x, D) u$ in place of $u$ in (30) and observing that

$$
\left\|P_{\circ}^{j}(D) f\right\| \leqq\|f\|_{2, k}, \quad f \in C_{\circ}^{\infty}(\Omega),
$$

we obtain

$$
\left\|P_{\circ}^{j}(D) E^{j} P^{j}(x, D) u\right\| \leqq(A B)^{j}\left\|P^{j}(x, D) u\right\| .
$$

Hence we conclude that

$$
\left\|P_{\circ}^{j}(D) u\right\| \leqq(A B)^{j}\left\|P^{j}(x, D) u\right\|, \quad u \in C_{\circ}^{\infty}(\Omega),
$$


because of (2), where $P_{\circ}$ is a $d$-hypoellptic polynomial.

On the other hand, if $v \in \Gamma^{\rho}(\Omega) \cap \mathscr{E}^{\prime}(\Omega)$, we have

$$
\left\|P^{j}(x, D) v\right\| \leqq C^{j+1}(j !)^{m \rho}, \quad j=1,2, \cdots,
$$

since the coefficients of $P(x, D)$ are in $\Gamma^{\rho}(\Omega)$.

By Theorem 2, conditions (31) and (32) imply that every solution $u \in C_{\circ}^{\infty}(\Omega)$ of equation (4) is in $\Gamma^{d \rho^{\prime}}(\Omega)$, when $v \in \Gamma^{\rho}(\Omega) \cap \mathscr{E}^{\prime}(\Omega)$. The theorem is thus established.

\section{REFERENCES}

1. L. Hörmander, Linear Partial Differential Operators, 4th ed., Springer-Verlag, 1976.

2.- On interior regularity of solutions of partial differential equations, Comm. Pure Appl. Math., 11 (1958), 197-218.

3. E. Newberger and Z. Zielezny, The growth of hypoelliptic polynomials and Gevrey classes, Proc. Amer. Math. Soc., 39 (1973), 547-552.

4. F. Trèves, Linear Partial Differential Equations with Constant Coefficients, Gordon and Breach, 1966.

Received May 31, 1978.

SUNY

Buffalo, NY 14214 


\section{PACIFIC JOURNAL OF MATHEMATICS}

\section{EDITORS}

DoNALD BABBITT (Managing Editor)

University of California

Los Angeles, California 90024

HUGo Rossi

University of Utah

Salt Lake City, UT 84112

C. C. MOORE and ANDREW OGG

University of California

Berkeley, CA 94720
J. DUGUNDJI

Department of Mathematics University of Southern Californı Los Angeles, California 90007

R. Finn and J. Milgram Stanford University Stanford, California 94305

\section{ASSOCIATE EDITORS}

E. F. BECKENBACH

B. H. Neumann
F. WOLF

K. YOSHIDA

\section{SUPPORTING INSTITUTIONS}

UNIVERSITY OF BRITISH COLUMBIA CALIFORNIA INSTITUTE OF TECHNOLOGY UNIVERSITY OF CALIFORNIA MONTANA STATE UNIVERSITY UNIVERSITY OF NEVADA, RENO NEW MEXICO STATE UNIVERSITY OREGON STATE UNIVERSITY UNIVERSITY OF OREGON
UNIVERSITY OF SOUTHERN CALIFORNIA STANFORD UNIVERSITY UNIVERSITY OF HAWAII UNIVERSITY OF TOKYO UNIVERSITY OF UTAH WASHINGTON STATE UNIVERSITY UNIVERSITY OF WASHINGTON 


\section{Pacific Journal of Mathematics}

Vol. 82, No. $1 \quad$ January, 1979

Werner Bäni, Subspaces of positive definite inner product spaces of countable dimension ...................................... 1

Marilyn Breen, The dimension of the kernel of a planar set..............

Kenneth Alfred Byrd, Right self-injective rings whose essential right ideals

are two-sided

Patrick Cousot and Radhia Cousot, Constructive versions of Tarski's fixed

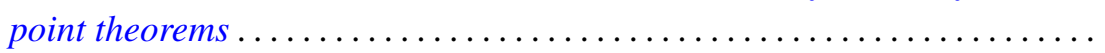

Ralph S. Freese, William A. Lampe and Walter Fuller Taylor, Congruence lattices of algebras of fixed similarity type. $I \ldots \ldots \ldots \ldots \ldots \ldots \ldots$

Cameron Gordon and Richard A. Litherland, On a theorem of Murasugi .....

Mauricio A. Gutiérrez, Concordance and homotopy. I. Fundamental

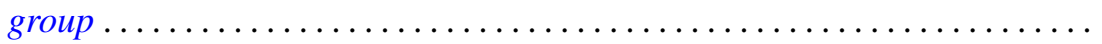

Richard I. Hartley, Metabelian representations of knot groups .............

Ted Hurley, Intersections of terms of polycentral series of free groups and free

Lie algebras ........................................

Roy Andrew Johnson, Some relationships between measures ............ 117

Oldřich Kowalski, On unitary automorphisms of solvable Lie algebras .......

Kee Yuen Lam, $K O$-equivalences and existence of nonsingular bilinear

maps...................................................

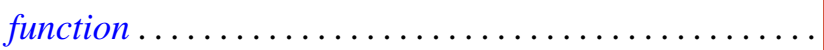

Robert A. Messer and Alden H. Wright, Embedding open 3-manifolds in compact 3-manifolds ............................

Gerald Ira Myerson, A combinatorial problem in finite fields. I . .

James Nelson, Jr. and Mohan S. Putcha, Word equations in a band of paths.

Baburao Govindrao Pachpatte and S. M. Singare, Discrete generalized Gronwall inequalities in three independent variables . .

William Lindall Paschke and Norberto Salinas, $C^{*}$-algebras associated with free products of groups ........................

Bruce Reznick, Banach spaces with polynomial norms ....

David Rusin, What is the probability that two elements of a finite group

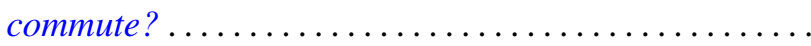

M. Shafii-Mousavi and Zbigniew Zielezny, On hypoelliptic differential operators of constant strength ...

Joseph Gail Stampfli, On selfadjoint derivation ranges .... . . .

Robert Charles Thompson, The case of equality in the matrix-valued triangle

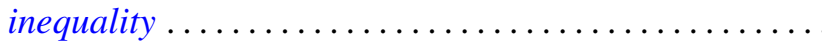

Marie Angela Vitulli, The obstruction of the formal moduli space in the negatively graded case. 